Check for updates

Cite this: Mater. Adv., 2022, 3,2558

Received 21st November 2021 Accepted 4th February 2022

DOI: 10.1039/d1ma01099a

rsc.li/materials-advances

\section{Polydiacetylene photocomposite material obtained by orthogonal chemistry: a detailed study at the mesoscopic scale $\dagger$}

\author{
Joan Teyssandier, (D) ${ }^{a}$ Marc Fouchier, (DD b Jacques Lalevée (iD *a and \\ Laurent Simon (D) *a
}

\begin{abstract}
A recent paper reported the spatially controlled photopolymerization and subsequent 3D printing of polydiacetylene (PDA) by orthogonal chemistry using dual-wavelength polymerization. Diacetylene monomers were dispersed in an acrylate resin to form a photocomposite in a two-step process: a first irradiation photopolymerizes the acrylate freezing the diacetylene monomers which were polymerized in a second step at a different wavelength. In the present article, for a better understanding of the organization of the generated functional composites, this process is studied at the mesoscopic scale by performing optical and scanning electron microscopy combined with correlative Raman, AFM and cathodoluminescence measurements. We have diluted the PCDA/acrylate blend in dichloromethane $\left(\mathrm{CH}_{2} \mathrm{Cl}_{2}\right)$ and performed drop casting deposition on graphite. We discovered that the acrylatediacetylene mixture promotes dramatically the formation of large PDA crystals. The confinement of PDA crystals inside the polyacrylate hindered their thermochromic blue-to-red transition, as revealed by correlative Raman microscopy. Cathodoluminescence measurements on the photocomposite have also shown that the light emission properties of PDAs are strongly modified by the induced confinement.
\end{abstract}

\section{Introduction}

Polydiacetylene (PDA) is one of the most studied photopolymers both concerning the fundamental aspects of its photopolymerization $^{1,2}$ and for its stimuli-responsive and light emission properties. ${ }^{3,4}$ It is a playground for the study of solidstate photopolymerization of monomeric crystals by topochemistry. The formation of PDA requires a precise molecular arrangement of its diacetylene (DA) monomers (where the diyne groups of the monomer are tilted by $45^{\circ}$ respectively to the expected axis of formation of the polymerized backbone and the adjacent diynes are distant by $3.5 \AA$ ). ${ }^{1}$ This order is achieved by a self-organization usually controlled by van der Waals interactions between the lateral chains of the monomer, leading to the formation of a supramolecular single crystal. The polymerization, which does not require a photoinitiator, leads to the formation of a quasi-perfect one-dimensional system. ${ }^{5}$

\footnotetext{
${ }^{a}$ Institut de Sciences des Matériaux de Mulhouse, CNRS-UMR 7361, Université de Haute Alsace, Mulhouse, France. E-mail: jacques.lalevee@uha.fr, laurent.simon@uha.fr

${ }^{b}$ Attolight AG, 1015 Lausanne, Switzerland

$\dagger$ Electronic supplementary information (ESI) available: Characterization of the samples at each step of the preparation by optical, electron and atomic force microscopies and Raman spectroscopy. Analysis of the samples on $\mathrm{SiO}_{2}$. Cathodoluminescence experiments. See DOI: 10.1039/d1ma01099a
}

PDA exists in two electronic structures called blue and red. PDA blue chains are planar and absorb light around $635 \mathrm{~nm}$, while red chains absorb around $530 \mathrm{~nm}$ and exhibit photoluminescence (PL), emitting at an energy of $2.3 \mathrm{eV}$ for isolated chain. ${ }^{6}$ The transition from blue to red is attributed to a finite torsion between subsequent monomeric units $\left(<20^{\circ}\right)^{7}$ leading to an indirect-to-direct bandgap transition by Brillouin zone folding (Peierls transition), which can be easily described by tightbinding calculations in the Hückel approximation. ${ }^{8}$ This limited finite torsion gives rise to an ordered red chain which has a lot of fascinating fundamental physical properties. Excitons with spatial coherence extending over macroscopic distances have for instance been observed in the case of ordered PDA red chains. ${ }^{3}$ In addition, this chromatic transition from nonfluorescence (blue phase) to strong fluorescence (red phase) can be triggered by various stimuli (such as temperature, $\mathrm{pH}$, mechanical stress...) making this material one of the most popular chromatic sensor. ${ }^{9-13}$ In the latter, the blue-to-red transition happens much more often by a non-finite rotation angle between subsequent monomer leading to an indirect-todirect bandgap transition by confinement due to the loss of $\pi$ electron delocalization. We call this red chain the "disordered" red chain. The blue-to-red transition and the possibility to control the reversibility of this transition is strongly dependent on the behavior and the conformation of the lateral alkyl chains 
attached to the central backbone..$^{12,14-16}$ In particular, the interactions occurring at the interface between these side chains and other compounds when the PDA is incorporated into a nanocomposite or nanostructured material have shown to influence drastically the reversibility and the conditions of the chromatic transition. ${ }^{14,17-19}$

This is a particularly relevant point considering that PDAs very often need to be combined with other materials in order to be used for its diverse applications. Shaping PDAs and controlling spatially their polymerization is for example a notably difficult challenge. In this context, a PDA-based photocomposite was recently used in additive manufacturing by leveraging the concept of orthogonal chemistry. By mixing PCDA (10,12pentacosadiynoic acid) monomers with a photopolymerizable triacrylate resin (TMPTA), it was possible to overcome the mechanical limitations of PDA and to photopolymerize it with spatial control. ${ }^{20}$ The polymerization happened in two steps: the polyacrylate was formed first by an irradiation at $405 \mathrm{~nm}$ using a focused laser beam (laser writing), then the PCDA monomers, that were frozen in the polyacrylate matrix, were photopolymerized using a UV lamp leading to the blue PDA phase. The latter underwent a blue-to-red transition by heating the sample at $80{ }^{\circ} \mathrm{C}$, showing the photocomposite retained the thermochromic properties of PDA. These macroscopic processes raise a lot of fundamental questions, such as how the PCDA monomers are dispersed in the (poly)acrylate matrix, how they self-assemble into monomeric crystals prior photopolymerization or about the possible role of phase separation in the TMPTA/PCDA mixture (and also about the origin of this phase separation: already existing in the monomer blend or induced by the photopolymerization reaction). The influence of the photopolymerization of the triacrylate resin on the selfassembly of PCDA is also still to investigate. But the most important questions probably concern the way the properties of PDAs are influenced by the polyacrylate-PDA interface.

To answer these questions, we study in the present paper this multistep formation process and the resulting properties of PDAs at the micro and nanoscale. We have prepared diluted mixtures of TMPTA and PCDA by using the same proportions of the molecular species used in the previous work ${ }^{20}$ but dissolved 100 times in $\mathrm{CH}_{2} \mathrm{Cl}_{2}$. The mixture is drop casted on HOPG samples and then irradiated and annealed. We have performed optical and scanning electron microscopy with correlative Raman, AFM and cathodoluminescence measurements.

\section{Experimental section}

\section{Materials}

Substrates consisted of highly oriented pyrolytic graphite (HOPG) and Silicon wafers with an oxide top layer. HOPG substrates were freshly cleaved before use. Si wafers were cleaned by successive ultrasonication in acetone and isopropanol (10 min each) followed by plasma cleaning at $80 \mathrm{~W}$ for 20 min (GV10x Asher instrument, ibss Group, Burllingame, USA). Trimethylolpropane triacrylate (TMPTA) and 1-benzyl-1- (dimethylamino)propyl-4-morpholinophenylketone (Irgacure 369) were obtained from Allnex and IGM, respectively. 10,12Pentacosadiynoic acid (PCDA) was obtained from Tokyo Chemical Industry ( $\geq 97 \%$, TCI-Europe) and Alfa Aesar $(\geq 98 \%)$. Dichloromethane (anhydrous, $\geq 99.8 \%$ ) was purchased from Sigma-Aldrich Co. LLC. PCDA was purified by filtering a solution the commercial product in dichloromethane and evaporate the solvent using a rotavapor. All other compounds were used as received.

\section{Samples preparation}

The composite was first prepared in the same proportions as in ref. 20, by dissolving PCDA (5\% in weight) and Irgacure 369 $(0.05 \%)$ in TMPTA. The composite was then dissolved 100 times in dichloromethane (unless otherwise stated) before depositing two droplets of the solution on HOPG or the Si wafers using a glass Pasteur pipette. After evaporation of the solvent, the films were sequentially polymerized in ambient conditions first under the irradiation of a violet LED centered at $405 \mathrm{~nm}$

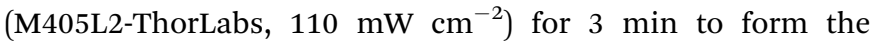
polyacrylate (poly-TMPTA) and in a second step using a polychromatic high-pressure mercury lamp (intelli-lamp Technology, Hamamatsu, $250 \mathrm{~mW} \mathrm{~cm}^{-2}$ ) for the UV-induced topotactic polymerization of PCDA monomers.

\section{AFM}

Atomic force microscopy measurements were performed in air with a Multimode AFM operating with a Nanoscope V controller (Bruker) in intermittent contact mode. Bruker (RTESPA-300) and NuNano (SCOUT 350) silicon cantilevers, with resonance frequency around $300 \mathrm{kHz}$ and spring constant around $40 \mathrm{~N} \mathrm{~m}^{-1}$, were used. Data analysis was performed using WSxM $5.0 .^{21}$

\section{SEM}

Scanning electron microscopy (SEM) was carried out either in a Philips XL30 FEG microscope (FEI-Thermo Fisher Scientific, Eindhoven, Netherlands) at an accelerating voltage of $2 \mathrm{kV}$ or a JEOL JSM-7900F microscope (Jeol, Tokyo, Japan) at an accelerating voltage of $1 \mathrm{kV}$. In order to preserve their electrical properties, the samples were observed as prepared, without a gold or carbon coating preparation.

\section{Raman}

Raman spectra and maps were acquired under ambient conditions with a Labram 300 spectrometer (Horiba) mounted on an Olympus BX40 confocal microscope. The $632 \mathrm{~nm}$ laser was selected to minimize the fluorescence background of the red phase of PDA. Raman spectra are resolved by a spectrometer using gratings of $600 \mathrm{~mm}^{-1}$, which leads to a spectral resolution of about $1.5 \mathrm{~cm}^{-1}$. For the annealing experiments, a heating stage (Linkam, THMS600) was used to heat the samples and temperatures were determined simply from the given values on the Linkam stage. The temperature on top of the samples surface may therefore be lower than indicated. The stage was heated at a ramp rate of $10{ }^{\circ} \mathrm{C} \mathrm{min}^{-1}$ and was 
subsequently maintained at the setpoint temperature for the duration of the measurements.

\section{Cathodoluminescence}

Cathodoluminescence (CL) measurements were performed with an Allalin SEM-CL microscope (Attolight AG, Switzerland) either at room temperature or $10 \mathrm{~K}$ and at an accelerating voltage of $3 \mathrm{kV}$.

\section{Results and discussion}

\section{Phase separation between PCDA and TMPTA}

As discussed in the introduction part, in order to understand how the PDAs are formed in such a matrix and how it affects its behavior, the mixture between PCDA and a TMPTA resin was studied here. The monomer composite was first prepared in the same proportions as in ref. 20, i.e. with 5\% (wt\%) of PCDA and $0.05 \%$ of the photoinitiator (Irgacure 369 ). To be able to study this system at the micro/nanoscale we have prepared thin films by dissolving the composite 100 times in $\mathrm{CH}_{2} \mathrm{Cl}_{2}$ before dropcasting the mixture on a highly oriented pyrolytic graphite (HOPG) substrate. Representative images of the film obtained by optical microscopy and SEM after deposition on HOPG and irradiation at $405 \mathrm{~nm}$ are shown in Fig. 1.

Fig. 1a shows a typical large scale SEM image where three different type of regions can be identified: droplet-like features of typically several tens of micrometers (such as those on the left side and the top right corner), apparently empty regions around the droplets and a multitude of smaller islands covering the rest of the surface (appearing with a bright contrast in Fig. 1a). The droplets consist of a polyacrylate matrix solidified after irradiation at $405 \mathrm{~nm}$ in which the PCDA in incorporated. PCDA crystals can be observed at the edge of the droplet in Fig. 1a (bright features). Raman mapping of the surface confirms that PCDA is present not only at the edges of the droplets but also all across them (see Fig. S4, ESI $\dagger$ ). The presence of PCDA crystals is confirmed in Fig. 1c, where structures with straight sides and sharp edges reveal crystalline order. The "empty" regions around the droplets are in fact covered by a thin layer of amorphous material probably composed by a mixture of PCDA and polyacrylate (see Fig S1, S3 and S5, ESI $\dagger$ ). They are the result of two phenomena: a dewetting due to the evaporation of the solvent $\left(\mathrm{CH}_{2} \mathrm{Cl}_{2}\right)$ and a shrinkage of the TMPTA during its photopolymerization into polyacrylate. The contribution of both phenomena will be discussed in a following section and in supporting information. The islands covering the rest of the HOPG surface are usually composed by one or several PCDA crystals. Some of these islands are shown in Fig. 1b, where it can be noticed that some crystals have a rectangular shape (they are bigger and exhibit a dark contrast in Fig. 1b) and some other appear as needles (the needles have a much brighter contrast in the image). Interestingly, the crystals are often embedded in small polyacrylate domains (Fig. S1-S3, ESI $\dagger$ ). Once again, the crystals can be unambiguously identified as PCDA by performing Raman mapping on these areas (see sections below and supporting information for more detail). Fig. 1c shows an optical micrograph of an area where a droplet and the islands present in its vicinity are separated by a very thin dewetting region. As opposed to SEM images, it is tricky to differentiate PCDA crystals and the polyacrylate in optical microscopy since they do not have a different contrast. It confirms however that PCDA crystals are typically present either above or below polyacrylate. It is also interesting to note that we do not observe PCDA crystals in regions apparently free of TMPTA/polyacrylate, such as the dewetting areas. This suggests a certain affinity between PCDA and the TMPTA resin but also a possible organization of PCDA domains due to the photopolymerization of TMPTA. Therefore, a too high solubility in a resin would also be detrimental to the formation of PCDA crystals since in that case the PCDA molecules would disperse homogenously in the resin which would thus prevent the adequate self-assembly of PCDA. It therefore seems that a subtle interplay is necessary to achieve the crystallization of PCDA in the acrylate resin and that the phase separation between the two components has a crucial role in this process. In any case this first observation shows that the chosen concentration (i.e. $5 \mathrm{wt} \%$ of PCDA in TMPTA) ${ }^{20}$ allows a first phase separation in the liquid resin leading to the
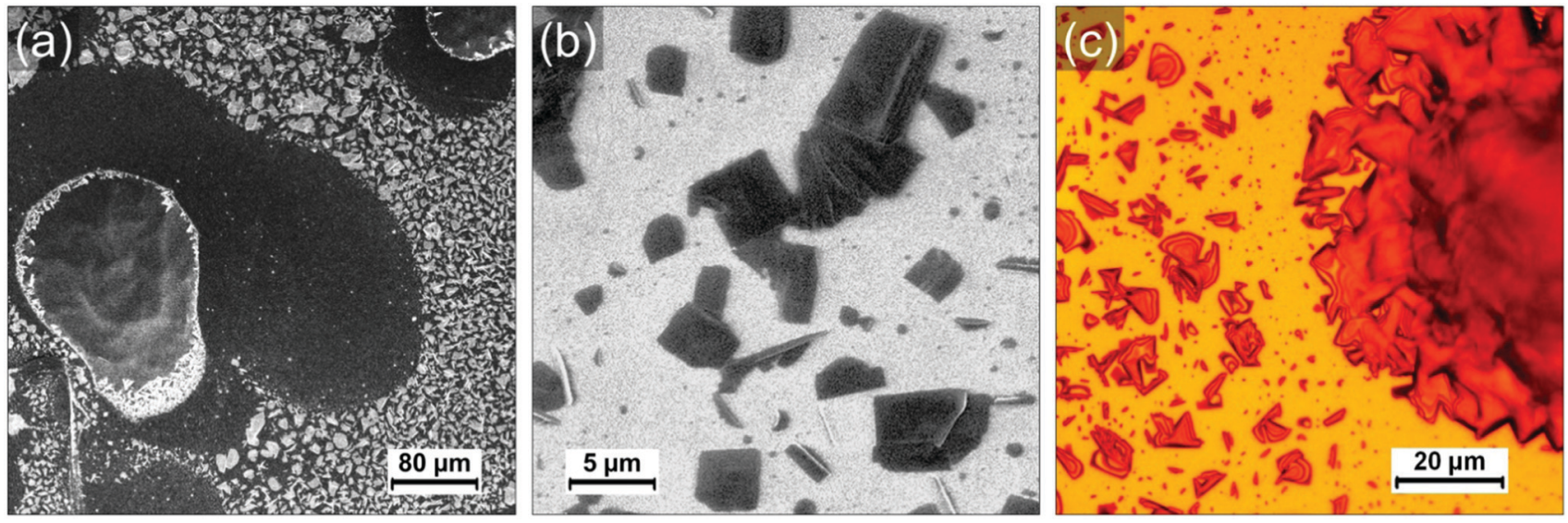

Fig. 1 (a and b) SEM images and (c) optical micrograph of the mixture (diluted 100 times) deposited on HOPG and irradiated 3 min at $405 \mathrm{~nm}$. 

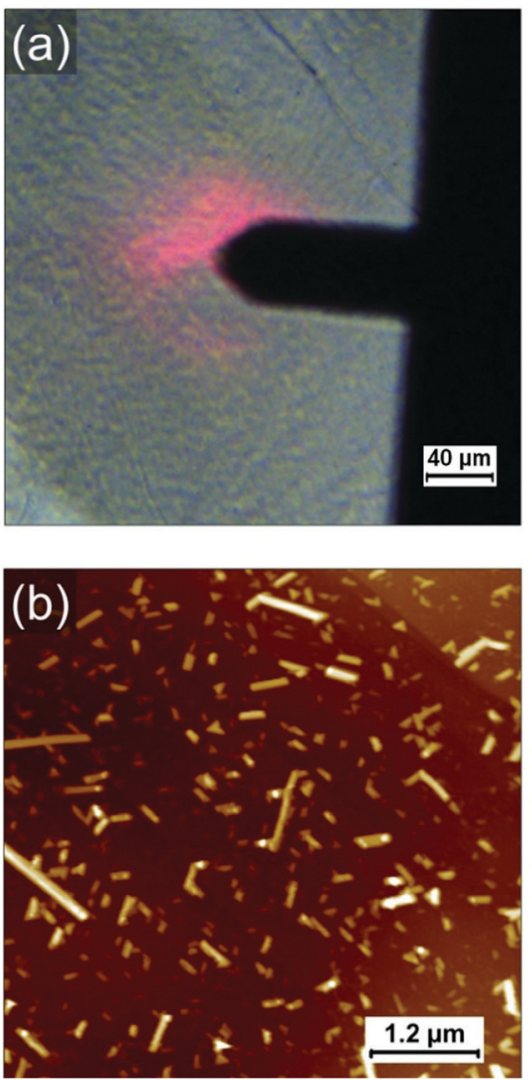

Fig. 2 Optical and AFM images of HOPG surfaces after deposition of solutions of (a and b) PCDA in $\mathrm{CH}_{2} \mathrm{Cl}_{2}\left(1.4 \times 10^{-3} \mathrm{~mol} \mathrm{L^{-1 }}\right)$ and (c and d) the mixture with equivalent concentration in PCDA (i.e. diluted 100 times). The AFM images in ( $\mathrm{b}$ and $\mathrm{d}$ ) correspond respectively to the region below the cantilever (in black) in (a) and to the red rectangle in (c).

self-organization of single crystal of monomers, which surprisingly favors the formation of large crystals.

To investigate the role of the (poly)acrylate resin in the formation of the PCDA domains, we have prepared and dropcasted a $1.4 \times 10^{-3} \mathrm{~mol} \mathrm{~L}^{-1}$ solution of pure PCDA in CH2Cl2 on HOPG and compared it with the drop-casting of a mixture solution with the same $1.4 \times 10^{-3} \mathrm{~mol} \mathrm{~L}^{-1}$ concentration of PCDA in TMPTA and $\mathrm{CH}_{2} \mathrm{Cl}_{2}$ (corresponding to a 100 times dilution of the formulation used in ref. 20). Both solutions were prepared just before deposition on the surface and the mixture was irradiated $3 \mathrm{~min}$ at $405 \mathrm{~nm}$ to polymerize the TMPTA. Since the quantity of photoinitiator (Irgacure 369) can be considered negligible, all the differences observed can be attributed to the presence of TMPTA and the photopolymerization process. PCDA crystals formed in absence of TMPTA are present with a high density and quite homogenously distributed on the surface, as evidenced by the AFM image in Fig. 2b. PCDA typically gives rise in this case to needles that generally extend over several tens of $\mathrm{nm}$ in width and a few hundreds of $\mathrm{nm}$ in length. The PCDA crystals formed after deposition from the TMPTA mixture are much bigger, as shown in Fig. $2 \mathrm{c}$ and d. They exhibit an important polydispersity in size, but often extend over one or several microns and have a rectangular shape. It is noticeable that these crystals exhibit a morphology and dimensions comparable to those obtained by the highest quality processes, such as the deposition under vacuum of evaporated monomers. ${ }^{22}$ This new result is really counterintuitive and confirms the role played by the TMPTA in assisting and guiding the crystallization of PCDA, probably driven by a phase separation.

The mixture has also been deposited on $\mathrm{SiO}_{2} / \mathrm{Si}$ wafer and similar observations were made (Fig. S7 and S8, ESI $\dagger$ ): large PCDA crystals are formed inside/on top of the polyacrylate. We conclude that the role of the surface is negligible in the selforganization process of PCDA and that only the solid-liquid phase separation in the mixture and the presence of TMPTA leads to such well-organized crystals of monomer.

\section{Effect of the polymerization processes}

In order to better understand the role of the photopolymerization in the formation of the PCDA crystals, a film of the photocomposite mixture has been prepared in sequential steps (drop-casting, irradiation at $405 \mathrm{~nm}$, irradiation in UV), and characterized between each of them. An image of the HOPG surface after deposition of the mixture and before any irradiation is shown in Fig. 3a. Several droplets of TMPTA can be observed. They are generally surrounded by a thinner liquid film. No PCDA crystal can be seen in the optical micrographs, either because no crystal big enough has been formed or because they are covered by the resin. However, AFM images 

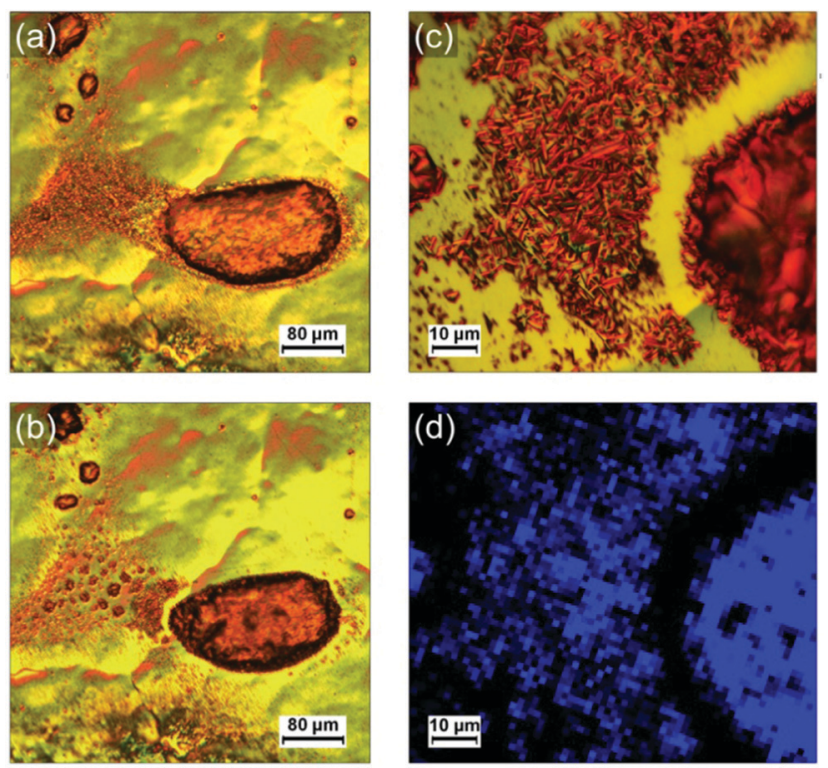

Fig. 3 Optical micrographs of the mixture (diluted 100 times) deposited on HOPG (a) before and ( $b$ and c) after irradiation during 3 min at $405 \mathrm{~nm}$. Image (c) corresponds to a zoomed-in region from (b). (d) Raman map of the density of polymerized blue PDA (i.e. intensity value of the Raman peak at $\approx 2080 \mathrm{~cm}^{-1}$ ). Map in (d) corresponds to the same region as in (c) after UV irradiation for $20 \mathrm{~s}$. Color code: black $=$ no PDA, blue $=$ PDA

performed on this film evidenced the existence of small rectangular PCDA crystals (Fig. S10, ESI $\dagger$ ). It shows that their crystallization already occurs (at least partly) in the liquid acrylate resin. Then, the sample is irradiated for $3 \mathrm{~min}$ at $405 \mathrm{~nm}$ to form the polyacrylate; this latter wavelength is quite selective to only activate photopolymerization of TMPTA. The image in Fig. 3b corresponds to the same area as in Fig. 3a after photopolymerization. The shrinkage of the droplets after irradiation is clearly observed, as well as the appearance of an "empty" strip between the biggest droplet and the thinner part of the film. Fig. 3c offers a zoomed image of such a region, revealing that the typical PCDA crystals (needles and rectangles) appear at the edges of the droplets, where the shrinkage is more pronounced. This observation is confirmed in other optical micrographs shown in Fig. S4 (ESI†). It suggests that the photopolymerization itself plays an important role in the self-organization and growth of the PCDA crystals.

A subsequent irradiation of the sample by UV light for a short time $(20 \mathrm{~s})$ does not result in any significant change in the morphology of the film confirming the vitrification of TMPTA at the first irradiation step. We only have noticed a slight reduction of the step edges height measured by AFM on the PCDA/PDA crystal surface from $5.1 \pm 0.4 \mathrm{~nm}$ before UV irradiation to $4.7 \pm 0.4 \mathrm{~nm}$ after. It suggests that the molecules are very slightly compressed after polymerization, something that has already been observed for PDAs. ${ }^{16}$ Based on the measured values these steps correspond to bilayers of vertically assembled PCDA molecules (see below for a more detailed discussion).

\section{Thermochromic properties of confined polydiacetylenes}

In addition to its influence on the crystallization of PCDA, it is important to elucidate how the polyacrylate matrix will impact the properties of the final PDAs. Indeed, since the side chains of PDAs are both of utmost importance for controlling their numerous properties and extremely sensitive to their environment, ${ }^{10-12,16}$ it is expected that the confinement in a matrix such as a polyacrylate will have an impact on the PDAbased nanocomposites made and patterned in this way. ${ }^{20}$ It was for instance shown in a previous work that the bulk PDApolyacrylate composite conserved its thermochromic properties while the blue-to-red transition usually observed at a high $\mathrm{pH}$ was inhibited. ${ }^{20}$ The thermochromic behavior of the nanocomposite film was therefore studied here as a model case. The polymerized mixture deposited on HOPG was heated up to a temperature of $75{ }^{\circ} \mathrm{C}$ for about $2 \mathrm{~h}$. Representative AFM images of PDA crystals before and after annealing are displayed in Fig. 4a and b, respectively. Both were obtained at room temperature.

AFM evidences how the morphology of the PDA crystals is impacted by the annealing. Numerous cracks appear within the crystal in Fig. $4 \mathrm{~b}$, all along its longitudinal axis. The rim of the crystal does not however undergo any change and the crystal conserves its original shape. Similar observations are typically made on all the crystals that have undergone a transformation after heating (see Fig. S15, ESI $\dagger$ ). Fig. 4c shows an AFM image obtained at room temperature of a rectangular PDA crystal from another sample (prepared with the exact same protocol) that was annealed at $95{ }^{\circ} \mathrm{C}$ for $5 \mathrm{~min}$. The cracks within this crystal are perfectly parallel to the longitudinal axis of the crystal. It appears also clearly that the rim of the crystal is different to the rest of the crystal. The rim appears unaffected by the cracks associated to the transition and seems amorphous. These rims typically exhibit a bright contrast in the phase image, similar to the phase contrast of bigger polyacrylate droplets (Fig. S15, ESI $\dagger$ ). Based on these observations, we attribute them to a thin polyacrylate region surrounding the PDA crystal. The empty space left by the cracks reveals an in-plane contraction of the crystal and it allows to measure the loss of area of PDA crystals. The average value obtained from eight crystals from several samples corresponds to a lateral contraction of $14 \pm 5 \%$. A similar feature has been observed in previous works when the PDAs undergo blue-to-red transition. ${ }^{23,24}$ A contraction of $28 \%$ has been for instance measured after a blue-to-red transition triggered in that case by an excess UV light exposure. ${ }^{24}$ This contraction and the consequent apparition of cracks in PDA layers (in that case, Langmuir films with PCDA molecules in upright conformation) was explained by a change in the angle between the side chains and the normal of the surface (from $35^{\circ}$ to close to $\left.0^{\circ}\right) .^{23,24}$ This interpretation was further supported by AFM measurements showing a $22 \%$ increase of the film thickness during the transition from the blue phase to the red phase. ${ }^{24}$ In our case, the thickness of the thinnest layers measured (step edges of the thinnest terraces) showed an 

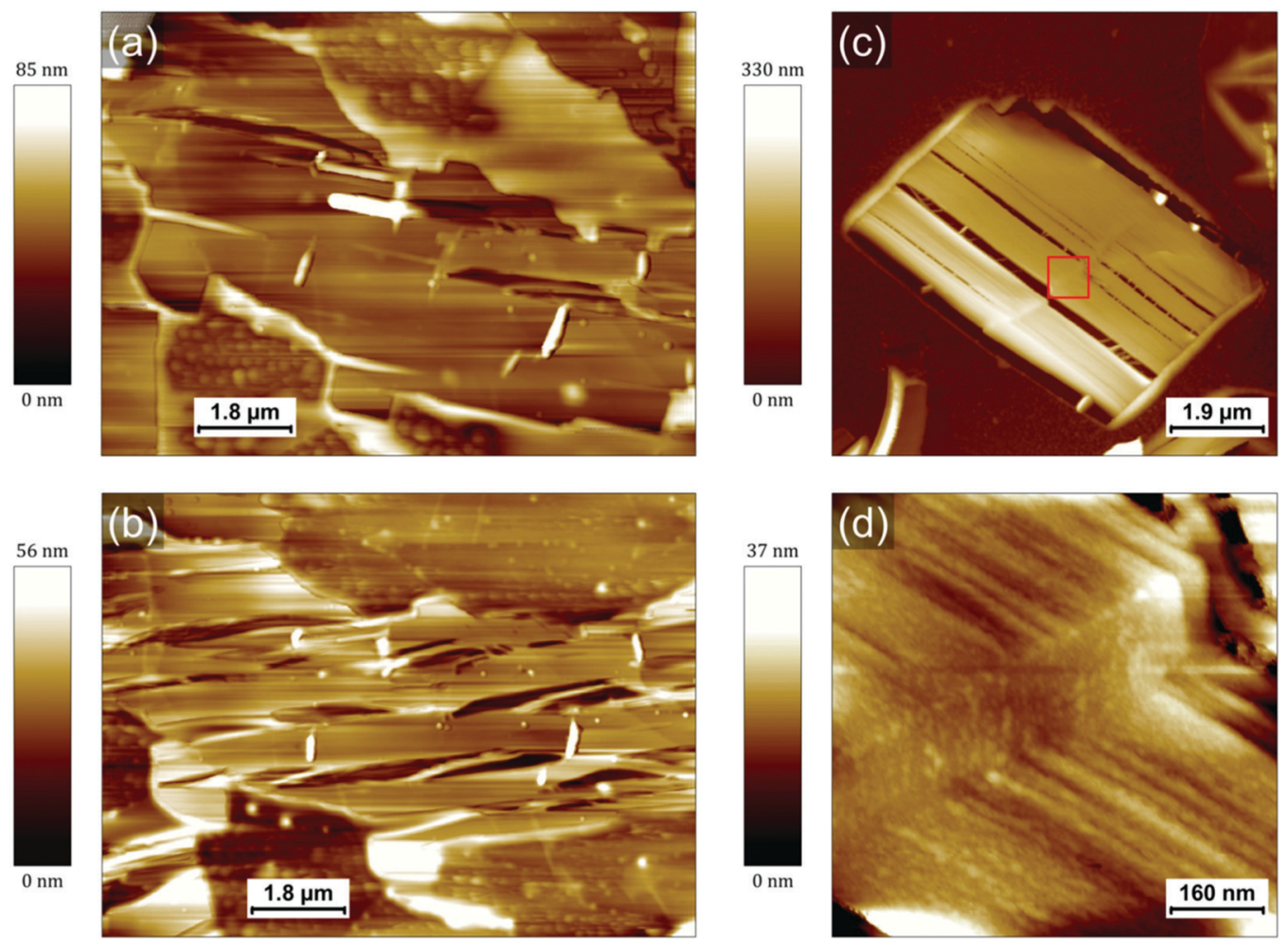

Fig. 4 AFM images of the same crystal of PDA (a) before and (b) after heating at $75{ }^{\circ} \mathrm{C}$ for $2 \mathrm{~h}$. (c and d) AFM image of another PDA crystal after annealing at $95^{\circ} \mathrm{C}$ for $5 \mathrm{~min}$. The AFM image in (d) correspond to the red rectangle in (c).

increase from $4.7 \pm 0.4 \mathrm{~nm}$ to $5.8 \pm 0.4 \mathrm{~nm}$ after annealing. It also confirms that the thinnest layers observed are bilayers based on comparison with literature ${ }^{16,24}$ and based on the length of an individual PCDA molecule $(3.2 \mathrm{~nm})$. The reason for this is that the initial self-assembly of PCDA is stabilized by hydrogen bonds between their carboxylic acid groups. It forms thus dimers that are stabilized within a layer by van der Waals interaction. The layers of the crystals before annealing are therefore $19 \pm 7 \%$ thinner than after, which is very consistent with the ref. 24 , and with a rotation from $\approx 35^{\circ}$ to $\approx 0^{\circ}$ (since $\left.\cos \left(35^{\circ}\right) \approx 0.82\right)$. This is therefore a first confirmation that the transformations observed after heating correspond indeed to a PDA blue-to-red transition. A higher resolution AFM image of the crystal (Fig. 4d) reveals that it is composed of fibers typically aligned along the longitudinal axis, as opposed to the flat terraces exhibited by the crystals before heating. The fibers are remarkably homogeneous in width, with values in the range $15-40 \mathrm{~nm}$, but more typically around $20 \mathrm{~nm}$. In such crystals, the PDA backbone is therefore oriented along the fibers with the side chains almost perpendicular to the graphite surface.

In order to study in more detail the blue-to-red transition occurring during heating, the samples have been analyzed by optical microscopy and correlative Raman spectroscopy. Fig. 5 shows typical objects observed on the sample after annealing and their corresponding Raman mapping. The blue and red phases of PDA are known to have different signatures in Raman (spectra of both phases are shown in Fig. 5a), ${ }^{20,25}$ allowing to identify and map them with sub-micron precision. By placing the samples on a heating stage, the thermochromic transition can be followed in situ during heating as well. The band at around $2100 \mathrm{~cm}^{-1}$ (corresponding to the $\nu(\mathrm{C} \equiv \mathrm{C})$ vibration) was used as a marker to follow the transition since there is no other peak in this region and because this is where the baseline is the flattest (the band at around $1500 \mathrm{~cm}^{-1}$ could alternatively be used). In this region, the blue phase exhibits a band at $\approx 2080 \mathrm{~cm}^{-1}$ that is shifted to $\approx 2120 \mathrm{~cm}^{-1}$ when the transition to the red phase occur. When the map is acquired, a full Raman spectrum is taken at every point (pixel size varying from $1 \mu \mathrm{m}$ to $5 \mu \mathrm{m}$ ). The maps are obtained by calculating for each point the difference between the intensities at $2080 \mathrm{~cm}^{-1}$ and $2120 \mathrm{~cm}^{-1}$. The pixel is colored as followed: blue or red dots correspond to areas where the PDA of this color is dominant. Purple dots correspond to areas where blue and red peaks are of similar intensities (i.e. either regions with similar amounts of both phases or regions without PDA). The results of in situ heating experiments can be found in supporting information (Fig. S12 and S13, ESI $\dagger$ ). One conclusion that can be drawn from it is that the blue-to-red transitions occurring in these PDA crystals are irreversible.

Optical microscopy images and the corresponding Raman maps taken at room temperature are shown in Fig. 5. They represent different objects observed on photocomposite films (diluted 100 times) on HOPG annealed respectively at $95{ }^{\circ} \mathrm{C}$ for 5 min (b and c) and $75{ }^{\circ} \mathrm{C}$ for $2 \mathrm{~h}$ (d and e). A larger crystal 
(a)
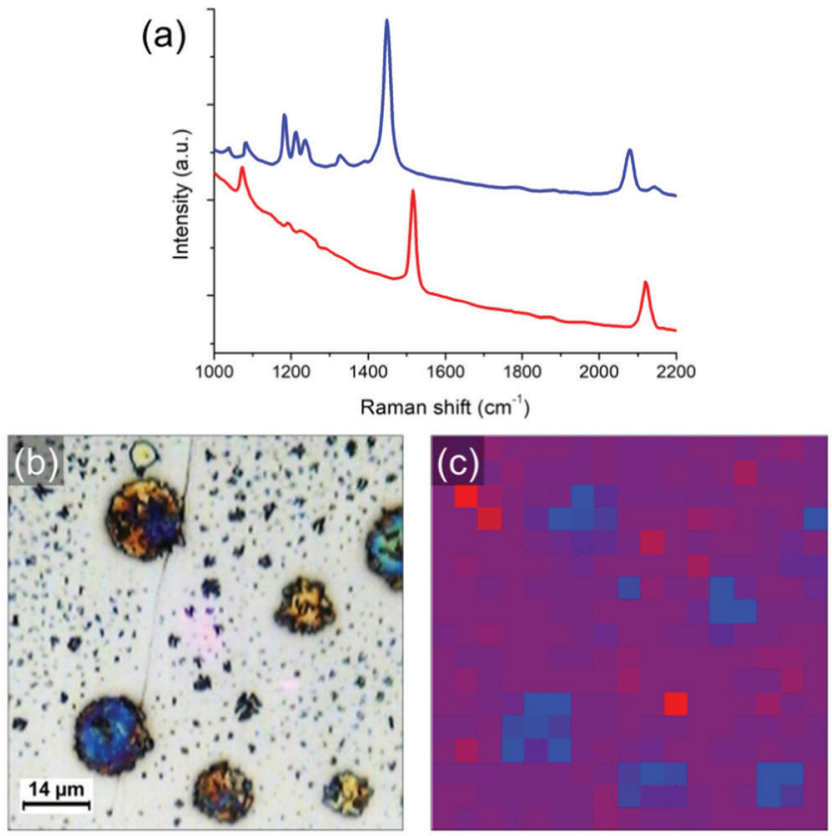
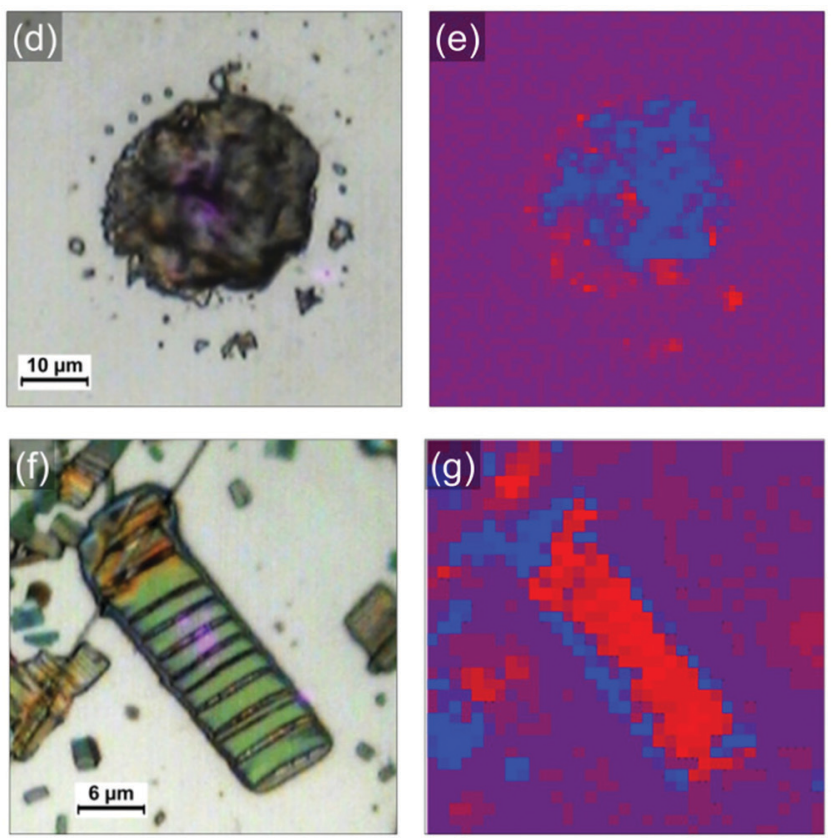

Fig. 5 (a) Raman spectra of the blue and red phases of PDA in the range $1000-2200 \mathrm{~cm}^{-1}$. (b, d and f) Optical micrographs and (c, e and g) corresponding Raman maps of typical structures observed on several sample after annealing at temperatures ranging from $75{ }^{\circ} \mathrm{C}$ ( $\mathrm{d}$ and $\mathrm{e}$ ) or $80{ }^{\circ} \mathrm{C}$ ( $\mathrm{f}$ and g) to $95{ }^{\circ} \mathrm{C}$ (b and c) (the transition temperature varied from sample to sample). Blue (red) pixels correspond to areas where the peak at $2080 \mathrm{~cm}^{-1}$ is higher (lower) than the one at $2120 \mathrm{~cm}^{-1}$ and purple ones to regions with either no PDA or with peaks at $2080 \mathrm{~cm}^{-1}$ and $2120 \mathrm{~cm}^{-1}$ of similar intensity. In ( $b$ and $c$ ) and ( $d$ and e) the circular objects correspond to PDA crystals incorporated in a thick polyacrylate droplet. These objects remained mostly blue after annealing while the "quasi polyacrylate-free" PDA crystals of smaller size located in the surrounding areas have undergone a blue-to-red transition. In the larger PDA island in ( $f$ and $g$ ), the bulk of the crystal became red whereas the edges covered by polyacrylate remain blue.

obtained from a more concentrated mixture solution (diluted 10 times) and annealed at $80{ }^{\circ} \mathrm{C}$ for $8 \mathrm{~min}$ is shown in Fig. $5 \mathrm{f}$ and g. Large droplets of polyacrylate with PDA crystals confined inside are presented in Fig. $5 \mathrm{~b}$ and d. A lot of small PDA crystals or aggregates of crystals are present around the droplets. These PDA crystals are almost free of polyacrylate. The inner part of the large crystal in Fig. $5 \mathrm{f}$ is free of polyacrylate while its edges are embedded and covered by a polyacrylate layer. The Raman maps in Fig. 5c, e and g identify which parts of these PDA objects have undergone the blue-to-red transition depending on the presence or not of polyacrylate. Raman maps confirmed that crystals with cracks are associated with the red phase of PDA (shifting of the $\nu(\mathrm{C} \equiv \mathrm{C})$ peak to $\left.\approx 2120 \mathrm{~cm}^{-1}\right)$, as illustrated with maps in Fig. $5 \mathrm{~g}$ and Fig. S14 (ESI $\dagger$ ). This shows that the reorganization of the chains observed in AFM corresponds indeed to the blue-to-red transition. An interesting feature observed on the samples after annealing is the tendency for polyacrylate-rich regions to exhibit the Raman signature of the blue phase of PDA. Blue PDAs are for instance present in majority in the polyacrylate "droplets" in Fig. $5 \mathrm{~b}$ and c while the rest of the PDA "free" crystals from the same image often exhibit Raman spectra of the red phase. A higher resolution Raman map of a single polyacrylate droplet is shown in Fig. 5e. Here it is clear that the inner part of the droplet, where the polyacrylate layer is thicker, remained predominantly blue. Conversely, the regions where the PDA is more "free" (i.e. not totally covered by the polyacrylate), on the edge and outside the droplet, exhibit almost only the signal of the PDA red phase. The crystal analyzed in Fig. 5f and g offers an even more striking evidence. Depositing a 10-times diluted solution (instead of 100 times) allowed the formation of bigger crystals, thus permitting to study with more accuracy the inhomogeneity of Raman signatures within the same crystal. The inner part of the crystal, which is polyacrylate-free, exhibits the typical cracks evidenced before in AFM images. It corresponds to a region of PDA red phase in the Raman map in Fig. 5g, confirming that the cracks are the consequence of the blue-to-red transition. Interestingly, the edges of the crystal seem unaffected by the cracks and resemble closely the structure observed by AFM in Fig. 4c. It corresponds therefore to a thin polyacrylate film surrounding the crystal. The Raman spectra of theses edges exhibit predominantly the peaks of the blue phase of PDA. This means that the PDA present in these regions, either confined within the polyacrylate or covered by it, have a temperature of chromatic transition higher than the free PDA crystals. This trend is confirmed by Raman maps in different regions and on different samples. We interpret this behavior by the existence of a mechanical stress due to the presence of polyacrylate above or around the PDA consequently hindering the mechanical relaxation in the blue-to-red transition. In other words, when crystals are confined in the hardened polyacrylate resin, the transition becomes more difficult.

\section{Correlative SEM and cathodoluminescence measurements}

Finally, the light emission properties of the photocomposite were analyzed. Correlative SEM and cathodoluminescence (CL) 

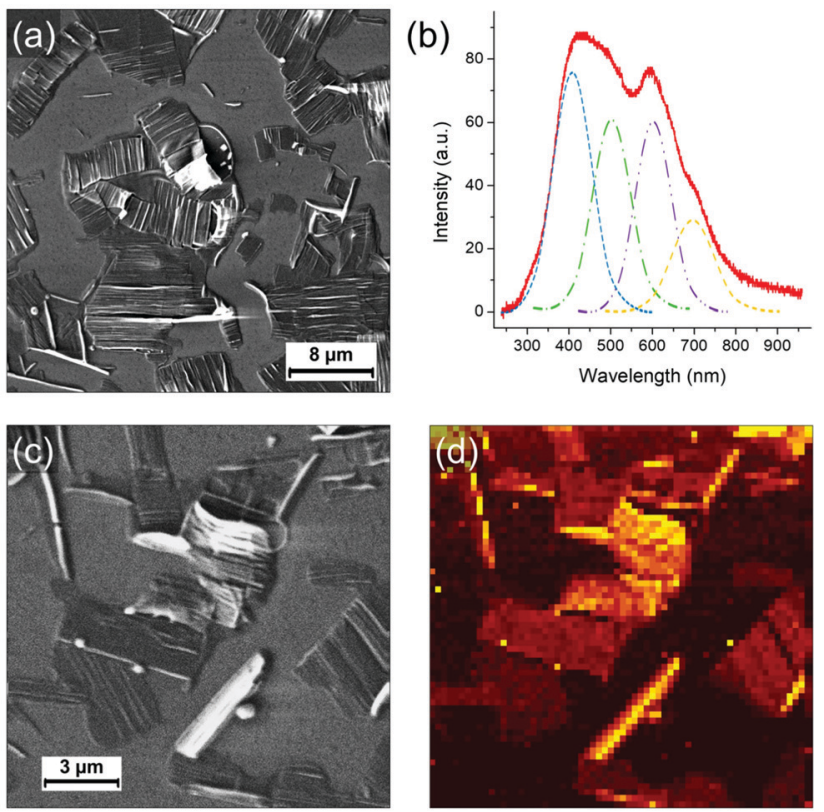

Fig. 6 Correlative cathodoluminescence $(C L)$ and SEM of PDAs on a sample of the mixture (diluted 10 times) deposited on HOPG after annealing at $80{ }^{\circ} \mathrm{C}$ for $8 \mathrm{~min}$ ( $\mathrm{CL}$ performed at room temperature and with a beam energy of $3 \mathrm{keV}$ ). (a) SEM images in a thin-film area with quasifree PDA crystals. (b) Average $\mathrm{CL}$ spectrum taken in this region. Correlative (c) SEM and (d) CL map of another thin region. The brightness of each pixel is proportional to the intensity of the signal of the $\mathrm{CL}$ spectrum taken at this point (integration over the entire wavelength range).

measurements were performed using an Allalin SEM-CL microscope from Attolight on the annealed $\left(80{ }^{\circ} \mathrm{C}\right.$ for $8 \mathrm{~min}$ ) sample of the mixture diluted 10 times and drop-casted on HOPG. The analysis was focused on two types of regions, respectively called "thin" and "thick". In the "thin" regions, PDA crystals are quasi "free" with little amount of polyacrylate whereas in the thick areas they are systematically confined or embedded in the solidified resin. As evidenced in the Raman maps in Fig. 5, the sample contains blue and red phases of PDA, with the blue phase more predominant in the polyacrylate-rich regions. CL consists in probing the emitted photons induced by the recombination of electron-hole pairs created by the electron irradiation from the SEM. As the kinetic energy of the electron is typically of few $\mathrm{keV}$, which considerably exceeds the bandgap of the material, the excitation promotes additional radiative and non-radiative transitions leading not only to the observation of a band associated to an exciton but also to all other vibronic excitons. In comparison to other spectroscopic techniques used to investigate PDAs, ${ }^{26}$ the main advantage of CL is that it allows to map the emission with a high spatial resolution.

Fig. 6 shows SEM images of a "thin" region. In this region, as discussed previously, the evaporation of the solvent and the shrinkage of the acrylate during polymerization releases large organized PDA crystals. As previously shown, they are not completely free of polyacrylate as they often remain embedded in polyacrylate (with the polyacrylate/PDA interface essentially present at the edge of the crystals). The CL spectrum in Fig. 6b, which is the average of the whole image, is typical of this region. We observe a broad band of emission between $300 \mathrm{~nm}$ and $800 \mathrm{~nm}$ in which four components can be deconvoluted at 409, 503, 602 and $698 \mathrm{~nm}$, respectively. The hyperspectral map of similar area (Fig. 6d) unambiguously shows that this broad band can be attributed to the PDA crystals. The bands at 503 and $602 \mathrm{~nm}$ can be attributed to the PDA crystal based on a comparison with literature. ${ }^{6,27}$ The band at $409 \mathrm{~nm}$ is possibly associated to the polyacrylate, which can also contribute partially to the band at $503 \mathrm{~nm}$ (the CL spectrum of the polyacrylate is shown in Fig. S16, ESI $\dagger$ ).

The case of the thicker regions of the sample is illustrated in Fig. 7. Correlative SEM image and colorized CL map are shown
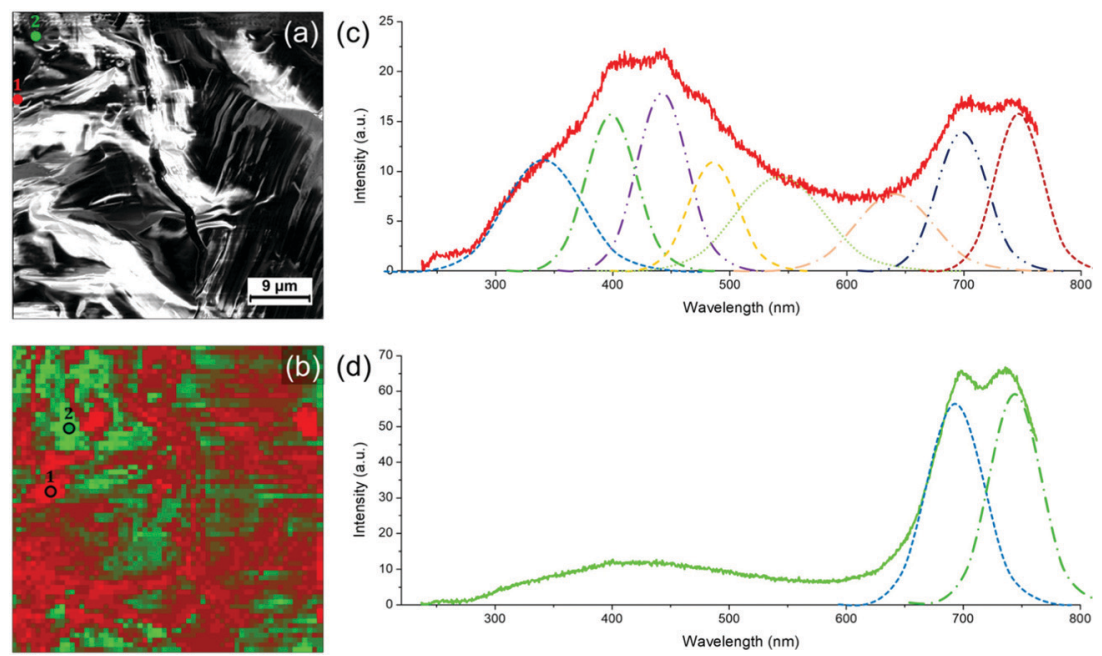

Fig. 7 Cathodoluminescence $(\mathrm{CL})$ of PDAs on a sample of the mixture (diluted 10 times) deposited on $\mathrm{HOPG}$ after annealing at $80{ }^{\circ} \mathrm{C}$ for 8 min $(\mathrm{CL}$ performed at $10 \mathrm{~K}$ and with a beam energy of $3 \mathrm{keV}$ ). (a) SEM image of a "thicker" region (PDAs crystal incorporated into a thick polyacrylate droplet). (b) Colorized CL map corresponding to the area in (a); red pixels correspond to spectra where the integrated $\mathrm{CL}$ intensity $<600 \mathrm{~nm}$ is superior to the intensity $>600 \mathrm{~nm}$, in the opposite case the pixel is green. (c) CL spectrum taken in point 1 (a and b). (d) CL spectrum taken in point 2 (a and b). 
in Fig. 7a and b, respectively. Two CL spectra (taken at $3 \mathrm{keV}$ and $10 \mathrm{~K}$ ) representative of the PDA signal in thick areas can be found in Fig. 7c and d. The spectrum in Fig. 7c exhibits a broad emission band between $250 \mathrm{~nm}$ and $800 \mathrm{~nm}$ where height emission bands can be deconvoluted. We observe the same emission bands at 543 and $641 \mathrm{~nm}$ as for the thin PDA crystals (including the possible contribution of the polyacrylate), with however a significant red shift. Both can be attributed to the exciton of red and blue phase, respectively, as they are in agreement with the values usually reported for these two PDA phases. $^{7}$ Other structures at lower wavelengths can be attributed to vibronic excitons but also, as previously discussed, to the polyacrylate. The band at $341 \mathrm{~nm}$ could possibly be due to continuum emission. We report two new structures above $650 \mathrm{~nm}$ : the one at $698 \mathrm{~nm}$, that was already observed in the thin film region (Fig. 6b), and the one at $747 \mathrm{~nm}$. In some areas of this CL map, these two bands are strongly enhanced, as illustrated by the spectrum in Fig. 7d, although the broad one between 300 and $600 \mathrm{~nm}$ as observed in Fig. 7c coexists. The two intense bands above $650 \mathrm{~nm}$ are only observed in the thicker regions of the photocomposite film, i.e. where the quantity of polyacrylate is more important. However, since the pure polyacrylate film does not emit at all at these wavelengths (Fig. S16, ESI $\dagger$ ), the comparison with this reference allows to attribute the two bands to PDA. We attribute these intense bands to the pressure induced by the polyacrylate film which confine the PDA crystal leading to the red shift of the two components observed at 543 and $641 \mathrm{~nm}$, respectively. Such an effect has never been observed so far on PDAs, but was evidenced by photoluminescence experiments under pressure on other organic crystals, such as for PTCDA. ${ }^{28}$

\section{Conclusions}

We performed a detailed, multi-technique study of the polymerization mechanisms at the micro- and nano-scale of a photocomposite material composed of two photopolymerizable monomers: a diacetylene derivative (PCDA) and a triacrylate resin (TMPTA). By preparing films of the photocomposite from diluted solutions in dichloromethane, we have directly evidenced the shrinkage of the TMPTA resin during the photopolymerization process. Counterintuitively, we have shown that the formation of large PCDA supramolecular crystal is strongly promoted in presence of the triacrylate monomer and probably driven by a phase separation mechanism. The dewetting process and the shrinkage of the TMPTA resin gave rise to two main types of crystals, some being completely confined into the polyacrylate matrix, some others being released from the (poly)acrylate droplets during the dewetting process (associated to the evaporation of the solvent) and the subsequent shrinkage of the polyacrylate during polymerization. In the latter case, the crystals are however often decorated by the polyacrylate at their edges, as evidenced by AFM measurement. Raman mapping measurements showed that the blue-to-red chromatic transition of the PDAs is hindered when they are confined in the polyacrylate and occurs thus at higher temperature. The influence of the polyacrylate on the properties of PDAs is moreover supported by cathodoluminescence experiments showing that the light emission properties of the PDAs are strongly modified by the confinement in the polyacrylate matrix. Other organizations of PDA structures driven by light can be investigated in forthcoming works.

\section{Author contributions}

J. T. and L. S. conceived the experiments. J. L. and L. S. supervised the project. J. T. prepared the samples, performed optical microscopy, AFM, SEM and Raman measurements and data analysis. M. F. performed cathodoluminescence measurements and data analysis. J. T. and L. S. wrote the paper with input from all the authors. All the authors read and discussed the paper.

\section{Conflicts of interest}

There are no conflicts to declare.

\section{Acknowledgements}

This work was supported by the CNRS, the Région Grand Est and FEDER (EU) through the NanoteraHertz project, and the French National Research Agency (ANR) through the MIXES project (Grant ANR-19-CE09-0028). J. T. and L. S. thank Thierry Barisien (INSP, Sorbonne Université) for fruitful discussions and Simon Gree for Raman measurments support.

\section{Notes and references}

1 R. H. Baughman, J. Appl. Phys., 1972, 43, 4362-4370.

2 V. M. Marina, O. Yuji, V. Elisseos, W. Kenji, T. Takashi, J. Christian and A. Masakazu, Nanotechnology, 2016, 27, 395303.

3 F. Dubin, R. Melet, T. Barisien, R. Grousson, L. Legrand, M. Schott and V. Voliotis, Nat. Phys., 2006, 2, 32-35.

4 J. Huo, Q. Deng, T. Fan, G. He, X. Hu, X. Hong, H. Chen, S. Luo, Z. Wang and D. Chen, Polym. Chem., 2017, 8, 7438-7445.

5 F. Dubin, J. Berréhar, R. Grousson, T. Guillet, C. Lapersonne-Meyer, M. Schott and V. Voliotis, Phys. Rev. B: Condens. Matter Mater. Phys., 2002, 66, 113202.

6 T. Barisien, L. Legrand, Z. Mu and S. Hameau, Phys. Chem. Chem. Phys., 2016, 18, 12928-12937.

7 A. M. Alvertis, R. Pandya, C. Quarti, L. Legrand, T. Barisien, B. Monserrat, A. J. Musser, A. Rao, A. W. Chin and D. Beljonne, J. Chem. Phys., 2020, 153, 084103.

8 C. Kollmar and H. Sixl, J. Chem. Phys., 1988, 88, 1343-1358. 9 X. Sun, T. Chen, S. Huang, L. Li and H. Peng, Chem. Soc. Rev., 2010, 39, 4244-4257.

10 X. Chen, G. Zhou, X. Peng and J. Yoon, Chem. Soc. Rev., 2012, 41, 4610-4630. 
11 S. Lee, J.-Y. Kim, X. Chen and J. Yoon, Chem. Commun., 2016, 52, 9178-9196.

12 X. Qian and B. Städler, Chem. Mater., 2019, 31, 1196-1222.

13 M. Weston, M. Ciftci, R. P. Kuchel, C. Boyer and R. Chandrawati, ACS Appl. Polym. Mater., 2020, 2, 5238-5248.

14 H. Peng, J. Tang, J. Pang, D. Chen, L. Yang, H. S. Ashbaugh, C. J. Brinker, Z. Yang and Y. Lu, J. Am. Chem. Soc., 2005, 127, 12782-12783.

15 D. J. Ahn, S. Lee and J.-M. Kim, Adv. Funct. Mater., 2009, 19, 1483-1496.

16 Y. K. Choi, S. Y. Lee and D. J. Ahn, J. Mater. Chem. C, 2019, 7, 13130-13138.

17 Y. Gu, W. Cao, L. Zhu, D. Chen and M. Jiang, Macromolecules, 2008, 41, 2299-2303.

18 A. Chanakul, N. Traiphol and R. Traiphol, J. Colloid Interface Sci., 2013, 389, 106-114.

19 K. Watanabe, H. Imai and Y. Oaki, Small, 2020, 16, 2004586.
20 Y.-Y. Xu, Z.-F. Ding, F.-Y. Liu, K. Sun, C. Dietlin, J. Lalevée and P. Xiao, ACS Appl. Mater. Interfaces, 2020, 12, 1658-1664.

21 I. Horcas, R. Fernández, J. M. Gómez-Rodríguez, J. Colchero, J. Gómez-Herrero and A. M. Baro, Rev. Sci. Instrum., 2007, 78, 013705.

22 Y. Yao, H. Dong, F. Liu, T. P. Russell and W. Hu, Adv. Mater., 2017, 29, 1701251.

23 A. Lio, A. Reichert, D. J. Ahn, J. O. Nagy, M. Salmeron and D. H. Charych, Langmuir, 1997, 13, 6524-6532.

24 D. Y. Sasaki, R. W. Carpick and A. R. Burns, J. Colloid Interface Sci., 2000, 229, 490-496.

25 K. Seto, Y. Hosoi and Y. Furukawa, Chem. Phys. Lett., 2007, 444, 328-332.

26 T. Barisien, L. Legrand, G. Weiser, J. Deschamps, M. Balog, B. Boury, S. G. Dutremez and M. Schott, Chem. Phys. Lett., 2007, 444, 309-313.

27 A. I. Gutman and A. V. Sel'kin, Phys. Lett. A, 1991, 158, 158-160.

28 R. Tallman, B. Weinstein, A. DeSilva and H. Wagner, Phys. Status Solidi B, 2004, 241, 3334-3338. 